\title{
Dengue vaccine breakthrough infections reveal properties of neutralizing antibodies linked to protection
}

\author{
Sandra Henein, ${ }^{1}$ Cameron Adams, ${ }^{1}$ Matthew Bonaparte, ${ }^{2}$ Janice M. Moser, ${ }^{3}$ Alina Munteanu, ${ }^{2}$ Ralph Baric, ${ }^{1,4}$ and Aravinda M. de Silva \\ 1Department of Microbiology and Immunology, University of North Carolina School of Medicine, Chapel Hill, North Carolina, USA. ${ }^{2}$ Sanofi Pasteur, Swiftwater, Pennsylvania, USA. ${ }^{3}$ Sanofi Pasteur, Orlando, \\ Florida, USA. ${ }^{4}$ Department of Epidemiology, Gillings School of Public Health, University of North Carolina, Chapel Hill, North Carolina, USA.
}

\begin{abstract}
The 4 serotypes of dengue virus (DENV1-4) are mosquito-borne flaviviruses that infect humans. Live attenuated tetravalent DENV vaccines are at different phases of clinical testing. DENV vaccine developers have relied on neutralizing antibodies (NAbs) as a correlate of protection. A leading tetravalent vaccine (Dengvaxia) stimulated NAbs to the 4 DENV serotypes, yet overall vaccine efficacy was low in children who were DENV seronegative at baseline before vaccination. We compared the properties of (a) NAbs induced by WT DENV1 or DENV3 infections, which are strongly correlated with protection from repeat infections, and (b) NAbs induced by Dengvaxia in individuals who subsequently experienced DENV1 or DENV3 breakthrough infections. WT infections induced NAbs that recognized epitopes unique (type specific) to each serotype, whereas the vaccine stimulated qualitatively different NAbs that recognized epitopes conserved (crossreactive) between serotypes. Our results indicate that, among children who were DENV-seronegative at baseline, unbalanced replication of the DENV type 4 vaccine component in the tetravalent vaccine stimulates Abs capable of crossneutralizing DENV1 and DENV3 in vitro, but not protecting in vivo. In DENV-seronegative individuals who are vaccinated, we propose that type-specific NAbs are a better correlate of protection than total levels of NAbs.
\end{abstract}

\section{Introduction}

The 4 dengue virus serotypes (DENV1-4) are mosquito-transmitted flaviviruses estimated to infect over 100 million people every year. (1) DENV infections stimulate neutralizing antibodies (NAbs) that are correlated with protection. Several DENV vaccines are at different stages of clinical development (2-4). While the development of DENV vaccines has been guided by the presence of NAbs as a correlate of protection, recent studies indicate that the presence of NAbs to the 4 serotypes after vaccination is not a reliable correlate of protection (5-7). We compared the properties of NAbs induced by WT DENV1 and DENV3 infections and a leading vaccine (Dengvaxia developed by Sanofi Pasteur) to improve our understanding of the properties of protective Abs.

A person infected with DENV for the first time (primary infection) develops a durable serotype-specific (TS) NAb response that is correlated with resistance to reinfection by the same serotype (2-4, 8). After a primary infection, people are susceptible to second infections with new serotypes. DENV serotype crossreactive (CR) Abs induced by primary infections have been linked to enhanced viral replication and more severe disease during secondary infections (9). Individuals who have recovered from secondary DENV infections develop new populations of serotype CR NAbs that are cor-

Authorship note: SH and CA contributed equally to this work.

Conflict of interest: MB, JMM, and AM are employees of Sanofi Pasteur, which developed Dengvaxia.

Copyright: () 2021, American Society for Clinical Investigation

Submitted: December 21, 2020; Accepted: May 13, 2021; Published: July 1, 2021

Reference information: J Clin Invest. 2021;131(13):e147066.

https://doi.org/10.1172/JCl147066. related with durable serotype crossprotective immunity $(4,10,11)$ To minimize the risk of DENV vaccines inducing Abs that enhance DENV infections, leading vaccines are based on tetravalent formulations to induce balanced protective immunity to all 4 serotypes.

Dengvaxia is a live attenuated chimeric tetravalent dengue vaccine (CYD-TDV) that was developed by modifying the yellow fever 17D live attenuated vaccine to contain the envelope (E) and premembrane proteins of each DENV serotype (12). The safety and efficacy of Dengvaxia was tested in a phase 2b trial (CYD23; ClinicalTrials. gov NCT00842530) in Thailand and 2 large phase 3 trials (CYD14, NCT01373281; CYD15, NCT01374516) in Asia and Latin America, respectively (13-16). Efficacy was high in children with preexisting immunity to DENVs who received the vaccine. In DENV-naive children, the vaccine reliably stimulated NAbs to the 4 serotypes, yet overall efficacy was low $(6,16)$. More recently, preliminary efficacy data from another clinical trial (TAK-003 developed Takeda, NCT02747927) also indicate that the presence of vaccine-induced $\mathrm{NAb}$ alone is not a reliable indicator of protection in children who were DENV seronegative at baseline (5). In individuals with no prior immunity to DENVs, we propose that TS NAbs directed to unique epitopes on each serotype are a better correlate of protection than total NAbs because the induction of TS Abs requires replication of the matched vaccine component $(17,18)$. To test this hypothesis, we characterized Dengvaxia-induced Ab responses in baseline seronegative individuals who subsequently experienced symptomatic breakthrough infections with DENV1 or DENV3. As controls, we characterized the properties of Abs in baseline seronegative vaccine recipients who did not experience a breakthrough infection and in individuals exposed to primary WT DENV1 or DENV3 infections who are protected from repeat infections by the same serotype. 
A

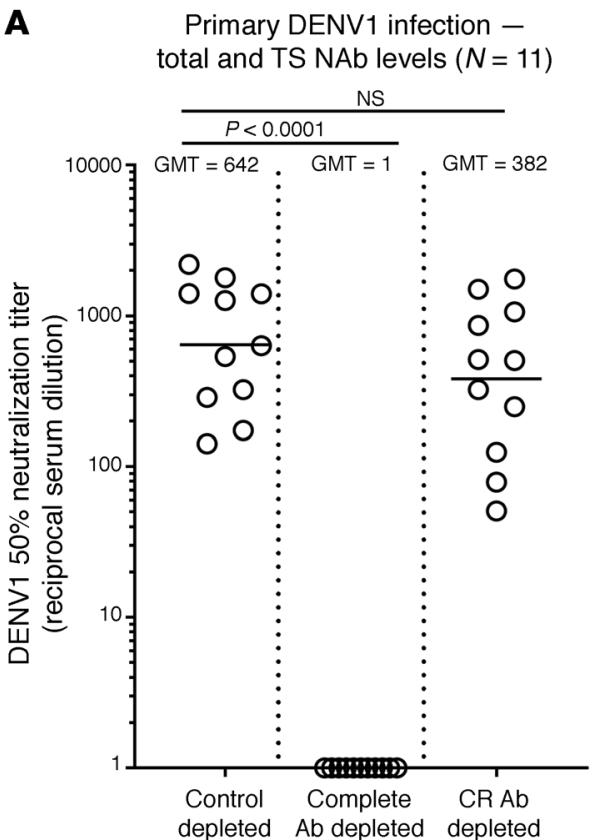

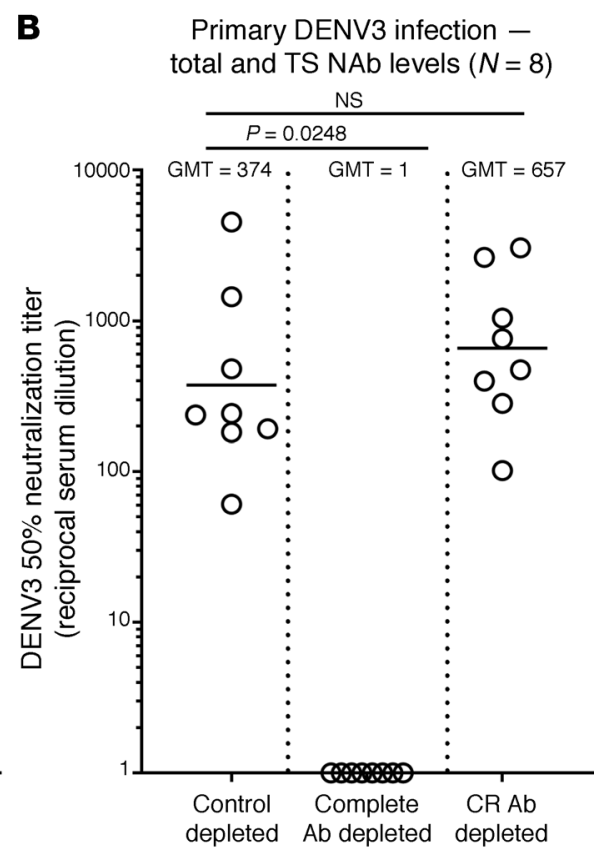

\begin{abstract}
Figure 1. Ab responses following primary DENV1 or DENV3 infection. Convalescent sera from people exposed to primary DENV1 (A) or DENV3 (B) infections were tested for NAb before (Control depleted) and after removal of total DENV Abs (Complete Ab depleted) or DENV serotype CR Abs (CR Ab depleted). Total Abs were depleted using DENV serotype responsible for infection as the depleting antigen (DENV1 or DENV3), and CR Abs were removed using a mix of antigen from heterologous DENV serotypes (DENV2 and DENV4 antigens for DENV1 immune sera and DENV1, DENV2, and DENV4 antigens for DENV3 immune sera). Levels of DENV1 or 3 neutralizing Abs were not significantly reduced by the removal of CR Abs. Friedman's 1-way ANOVA was used to establish statistical significance.
\end{abstract}

\section{Results and Discussion}

$A b$ response to WT DENV1 or DENV3 infections. We characterized the specificity of NAbs in individuals who experienced primary DENV1 $(n=11)$ or DENV3 $(n=8)$ infections (Supplemental Table 1; supplemental material available online with this article; https://doi.org/10.1172/JCI147066DS1) because WT infections are known to induce durable protection from clinically symptomatic reinfection with the same serotype $(2,4)$. To measure levels of DENV1 or DENV3 TS Abs, we incubated the samples with beads coated with DENV serotypes not responsible for infection to deplete serotype CR Abs (Supplemental Figure 1). The depleted samples bound to DENV1 or DENV3, although at reduced levels compared with control depleted samples, demonstrating the presence of TS Abs to each serotype responsible for infection (Supplemental Figure 1). The CR Ab depleted samples neutralized the homologous serotype of infection (DENV1 or DENV3) at levels comparable to those of control depleted samples, demonstrating that TS Abs were mainly responsible for functional neutralization (Figure 1, A and B).

Dengue vaccine responses in children who experienced DENV1 or DENV3 breakthrough infections. Most children with no immunity to DENVs who received Dengvaxia developed NAbs to DENV1 and DENV3, yet vaccine efficacy against these 2 serotypes was low (6). We characterized the properties of vaccine-induced Abs in baseline seronegative children who subsequently experienced DENV1 ( $n=15$ subjects) or DENV3 ( $n=18$ subjects) breakthrough infections (Supplemental Table 2). When vaccine immune sera from DENV1 breakthrough cases were depleted of CR Abs, we observed that nearly all DENV1 binding was due to CR Abs, whereas all subjects had DENV4 TS binding Abs (Supplemental Figure 2A). A similar pattern was observed when the $\mathrm{Ab}$-depleted samples were tested for neutralization of DENV1 and DENV4 (Figure 2). While all 15 subjects who experienced DENV1 breakthrough infections had developed DENV1 NAbs after vaccination (geometric mean titer [GMT] $=97$ ), only 4 of 15 subjects had any DENV1 TS NAbs (GMT = 2.5; Figure 2, A and C, and Supplemental Figure 4A). In contrast, 10 of 15 children had DENV4 TS NAbs (GMT = 15; Figure 2, B and C, and Supplemental Figure 4A).

Among the 18 children who experienced DENV3 breakthrough infections, 14 had DENV3 NAbs (GMT =28) and all 18 had DENV4 NAbs (GMT = 122; Figure 3A). After Ab depletion to remove CR Abs, only 4 of 18 subjects had DENV3 TS NAbs (GMT= 2) and 14 of 18 subjects had DENV4 TS NAbs (GMT = 19; Figure 3, B and C, and Supplemental Figure 4B). Dengvaxia mainly stimulated DENV1 and DENV3 CR NAbs in children who subsequently experienced DENV1 or DENV3 breakthrough infections.

Dengue vaccine responses in the general population of children who were seronegative at baseline. Next, we characterized the properties of DENV1, DENV3, and DENV4 NAbs in baseline seronegative vaccine recipients $(n=11)$ who did not experience a breakthrough infection during the clinical trial. These subjects had variable levels of NAbs to the 3 serotypes tested, with the highest response to DENV4 followed by DENV3 then DENV1 (GMT=103, 67, and 60 , respectively; Supplemental Figure 3). When the sera were subjected to Ab depletions to estimate levels of TS NAbs, we observed that 2 of 11 had DENV1 TS NAbs, 3 of 11 had DENV3 TS NAbs, and 5 of 11 had DENV4 TS NAbs (Supplemental Figure 3). As the breakthrough and nonbreakthrough cases had similar Ab profiles, we combined the results from all the children tested to define properties of DENV1, DENV3, and DENV4 Abs stimulated by the vaccine in children who were seronegative at baseline (Figure 4). Although nearly all children (42 of 44) had DENV1 NAbs, only 12 (27\%) had DENV1 TS NAbs (Figure 4, A and D). Similarly, 24 of 29 children tested had DENV3 NAbs, but only 7 (24\%) had DENV3 TS NAbs (Figure 4, B and D). In contrast with what occurred with DENV1 and DENV3, 28 of 44 (64\%) children tested had TS NAbs to DENV4 (Figure 4, C and D). 
A

DENV1 vaccine breakthrough cases $(N=15)$ DENV1 total and TS NAb levels

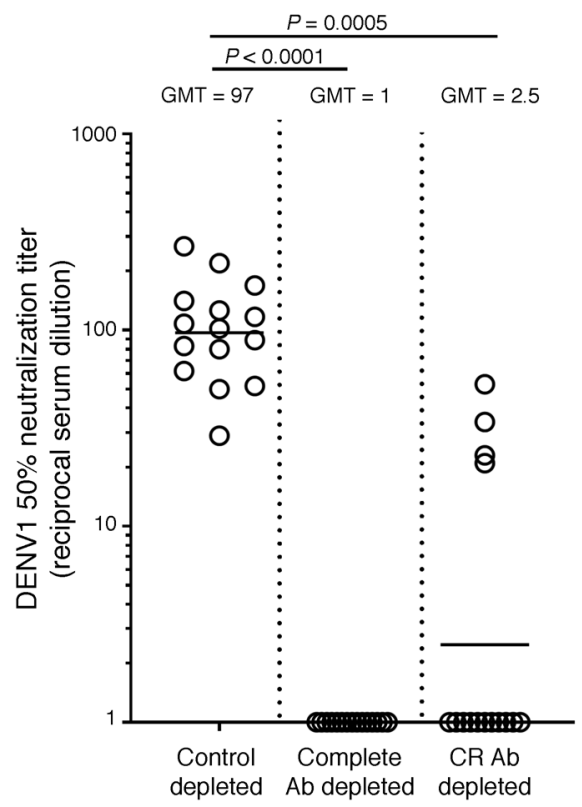

B

DENV1 vaccine breakthrough cases $(N=15)$ DENV4 total and TS NAb levels

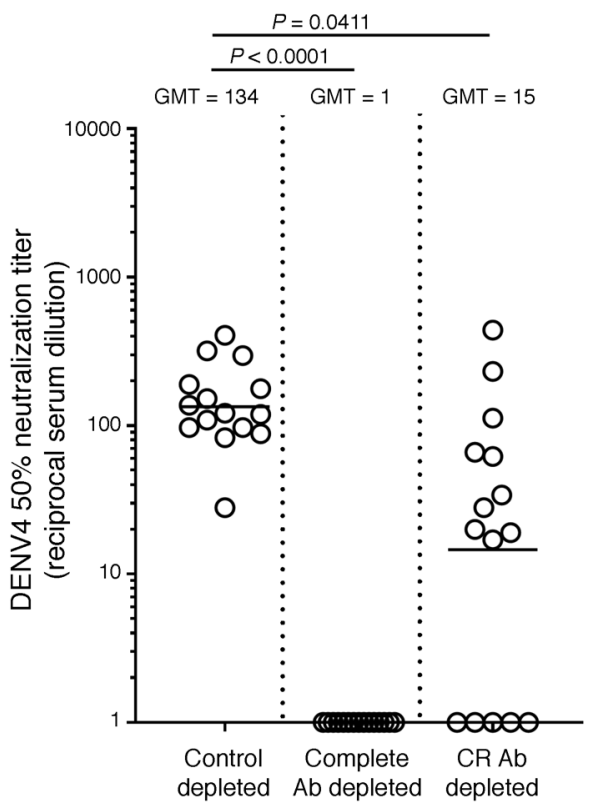

C

DENV1 vaccine breakthrough cases $(N=15)$ $\%$ of DENV 1 and DENV4 TS NAb

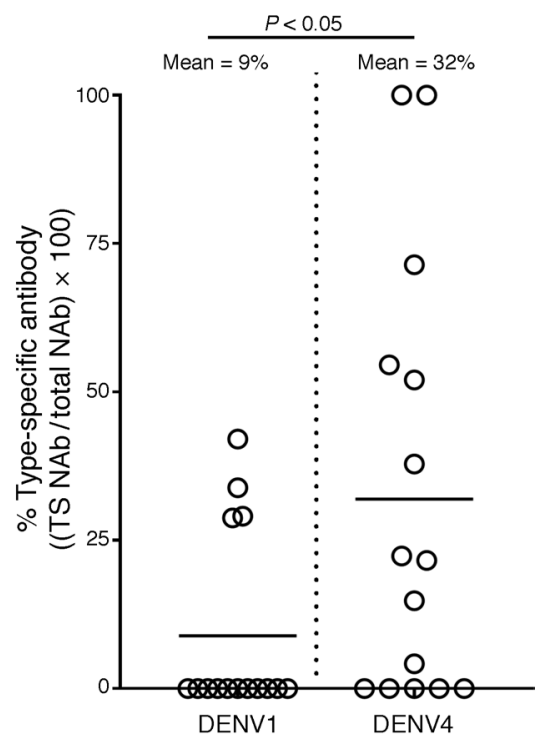

Figure 2. Vaccine-induced Ab responses in children who experienced DENV1 breakthrough infections. Vaccine responses to DENV1 (A) and DENV4 (B) were analyzed in baseline seronegative children who received Dengvaxia and subsequently experienced DENV1 $(n=15)$ breakthrough infections. (A) DENV1 NAb responses after vaccination were measured without depleting any Ab (Control depleted), after removal of all DENV1-binding Abs (Complete Ab depleted with DENV1 antigen), and after removal of CR Ab (CR Ab depleted using a mix of DENV2 and DENV4 antigens). (B) DENV4 $\mathrm{NAb}$ responses after vaccination were measured without depleting any Ab (Control depleted), after removal of all DENV4-binding Ab (Complete Ab depleted with a mix of DENV2 and DENV4 antigens), and after removal of CR Ab (CR Ab depleted using DENV1 antigen). (C) The data were also analyzed to compare the percentages of DENV1 and DENV4 TS NAbs in the children after vaccination. Friedman's 1-way ANOVA test (A and B) or Wilcoxon's signed ranks test (C) was used to establish statistical significance.

The clinical observation that Dengvaxia stimulated NAbs in baseline seronegative individuals that were not correlated with protection was unexpected because NAbs are correlated with the success of other flavivirus vaccines. The aim of our study was to identify improved $\mathrm{Ab}$ correlates of protection against DENV1 and DENV3 by comparing the properties of Abs in vaccine recipients who experienced breakthrough infections and individuals exposed to primary WT DENV1 and DENV3 infections. Our results demonstrate that both WT DENV infection and Dengvaxia stimulated NAbs to DENV1 and DENV3, but NAbs stimulated by WT virus infections targeted epitopes unique to DENV1 or DENV3, whereas the vaccine mainly induced NAbs to epitopes conserved between serotypes. In contrast to the DENV1 and DENV3 responses, most children developed DENV4 TS NAbs after vaccination. These results match our previous observation that adults immunized with Dengvaxia mainly develop TS NAbs to DENV4 and CR NAbs to other serotypes (19). The DENV4 component in Dengvaxia replicates to higher levels and/or infects more individuals than the other 3 components $(12,20,21)$. We propose that in individuals with no preexisting immunity to DENVs, the robust replication of the DENV4 vaccine component stimulates serotype CR Abs that neutralize DENV1 and DENV3 in cell culture, but these Abs are not protective or correlated with protection in vivo.

At first glance, our conclusion that total levels of NAbs are a poor correlate of vaccine efficacy in baseline seronegative individuals appears to be inconsistent with recent publications demonstrating that levels of NAbs induced by Dengvaxia are correlated with vaccine efficacy $(7,22)$. However, these reports are based on a pooled analysis of a group of baseline seropositive and seronegative children that mainly consisted of seropositive children. When the analysis was stratified by baseline serostatus, the correlation was weak and imprecise in baseline seronegative children $(7,22)$. These 2 populations require separate consideration when evaluating immune correlates, vaccine efficacy, and safety, given fundamental differences in how adaptive immunity is activated in individuals who are seronegative and seropositive at the time of vaccination.

Recent studies provide explanations for why some Abs might neutralize DENVs in cell culture, but not protect in vivo (23). DENV stocks produced using laboratory cell lines consist of virions at different stages of maturation with low specific infectivity (23). DENV1 virions circulating in humans were observed to be more mature and infectious than cell culture-derived virus used in cell culture neutralization assays (23). DENV1 TS mAbs and sera from people exposed to primary DENV1 infections neutralized both plasma- and cell culture-produced DENV1 efficiently (23). DENV serotype CR mAbs and heterotypic immune sera from people exposed to primary DENV2 or DENV3 infections efficiently neutralized cell culture-produced DENV1 and poorly neutralized or failed to neutralize human plasma-derived DENV1(23). 
A

DENV3 vaccine breakthrough cases $(N=18)$ DENV3 total and TS NAb levels

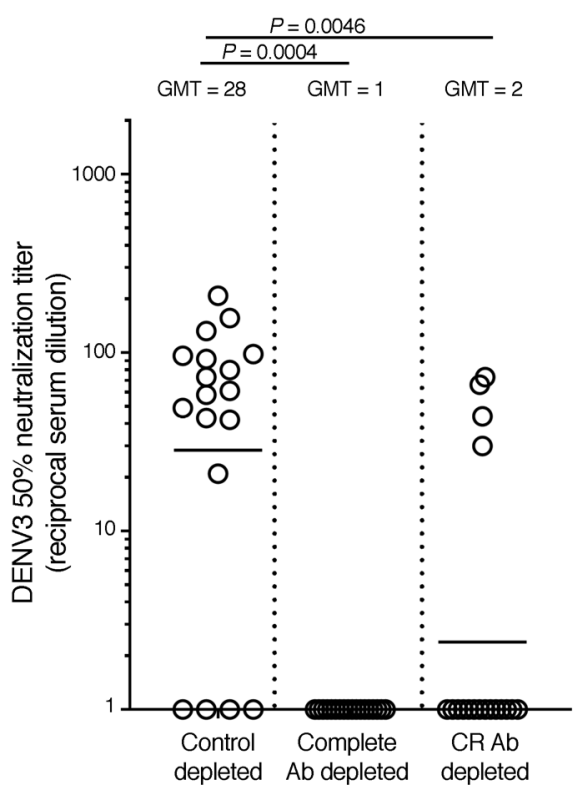

B

DENV3 vaccine breakthrough cases $(N=18)$ DENV4 total and TS NAb levels

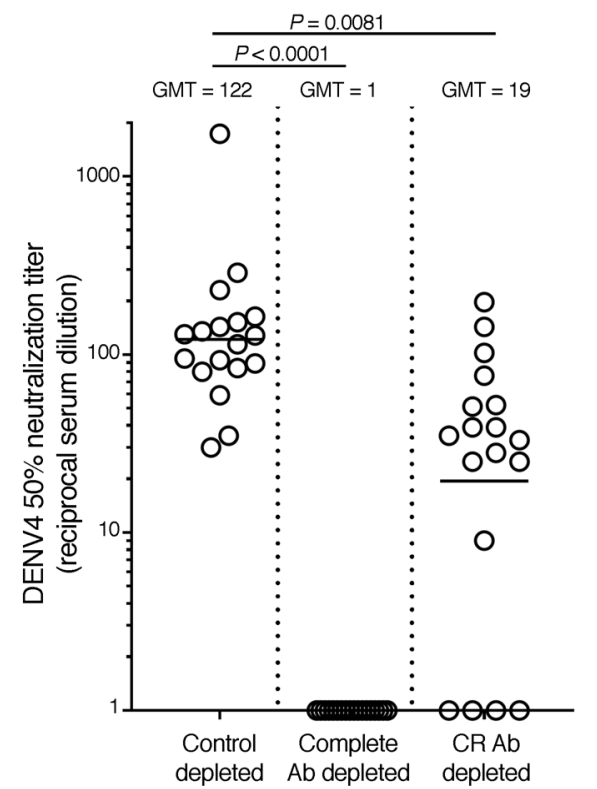

C DENV3 vaccine breakthrough cases $(N=18)$

$\%$ of DENV1 and DENV4 TS NAb

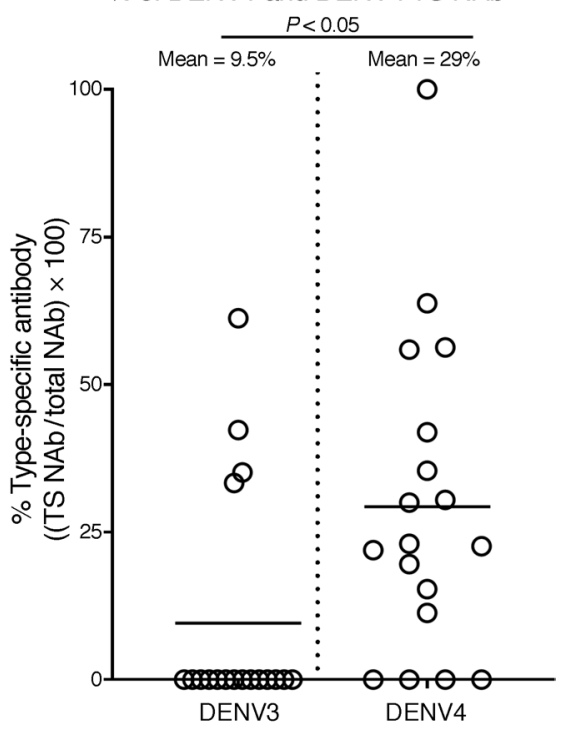

Figure 3. Vaccine-induced Ab responses in children who experienced DENV3 breakthrough infections. Vaccine responses were analyzed in baseline seronegative children who received Dengvaxia and subsequently experienced DENV3 $(n=18)$ breakthrough infections. (A) DENV3 NAb responses after vaccination were measured without depleting any Ab (Control depleted), after removal of all DENV3-binding Abs (Complete Ab depleted with DENV3 antigen), and after removal of CR Ab (CR Ab depleted using a mix of DENV1, DENV2, and DENV4 antigens). (B) DENV4 NAb responses after vaccination were measured without depleting any $\mathrm{Ab}$ (Control depleted), after removal of all DENV4-binding Abs (Complete Ab depleted with a mix of DENV1, DENV2, and DENV4 antigens), and after removal of CR Ab (CR Ab depleted using DENV3 antigen). (C) The data were also analyzed to compare the percentages of DENV3 and DENV4 TS NAbs in the children after vaccination. Friedman's 1-way ANOVA test (A and B) or Wilcoxon's signed ranks test (C) was used to establish statistical significance.

Most human DENV serotype-crossreactive Abs target conserved epitopes at or near the fusion loop at the tip of domain II of E protein. This region of $\mathrm{E}$ protein is partially exposed and accessible to $\mathrm{Ab}$ binding in immature virions, whereas in mature virions, the fusion loop is buried and not readily accessible to Ab binding (24, 25). DENV neutralizing Ab assays utilizing partially mature DENV stocks will measure NAbs to exposed and hidden epitopes on mature virions and overestimate levels of protective Abs.

For Dengvaxia, no significant efficacy against DENV1 or DENV3 was observed in dengue-seronegative children who were vaccinated and monitored for 5 to 6 years (6). Therefore, our observation that baseline seronegative vaccinated individuals had similar $\mathrm{Ab}$ profiles in the breakthrough and nonbreakthrough groups is not surprising. In the absence of subgroups within the seronegative vaccinated population for identifying immune correlates, we compared $\mathrm{Ab}$ responses in individuals exposed to primary DENV1 and DENV3 infections and individuals who were vaccinated to identify improved correlates. While not the perfect comparison, our finding that DENV1 and DENV3 TS NAbs are readily detected after natural infection and rarely in baseline seronegative vaccinated children who experienced breakthrough infections indicates that TS NAbs are a more reliable correlate than total levels of NAbs to guide the development of DENV vaccines for use in this population.

We note that a few vaccinated individuals who developed low levels of TS NAbs to DENV1 or DENV3 experienced breakthrough infections with the matched serotype. Therefore, the presence of TS NAbs alone may not always be sufficient for protection. TS
NAbs may have to be maintained above a certain threshold for protection. Individuals with TS NAb responses may be susceptible to infections caused by natural variant strains that differ at critical TS Ab epitopes from the vaccine strain.

In the current study, we focused on Ab responses to DENV1 andDENV3 in seronegative children who received Dengvaxia. In this population, the vaccine was also not efficacious against DENV2, while the clinical trials demonstrated significant efficacy against DENV4 (16). Studies are currently in progress so that we can better understand vaccine responses to DENV2 and DENV4. Dengvaxia was efficacious in children with preexisting immunity to DENVs who were vaccinated, and our study was not designed to identify vaccine correlates and mechanisms of protection in this population. In individuals with no baseline immunity to DENVs, we propose that TS NAbs are a better correlate of protection than the current standard of total NAbs.

\section{Methods}

Detailed Methods appear in Supplemental Methods.

Statistics. Friedman's 1-way ANOVA test was used to establish statistical significance between the control depleted samples and the $\mathrm{Ab}$ depleted ones. Comparisons of percentages of serotype-specific and absolute serotype-specific neutralization titers were done by Wilcoxon's signed ranks test (Figure 2 and Figure 3) and the Kruskal-Wallis test (Figure 4).

Study approval. The CYD14 (ClinicalTrials.gov NCT01373281) and CYD15 (ClinicalTrials.gov NCT01374516) trial protocols have been approved by all relevant ethics review boards $(26,27)$. The 

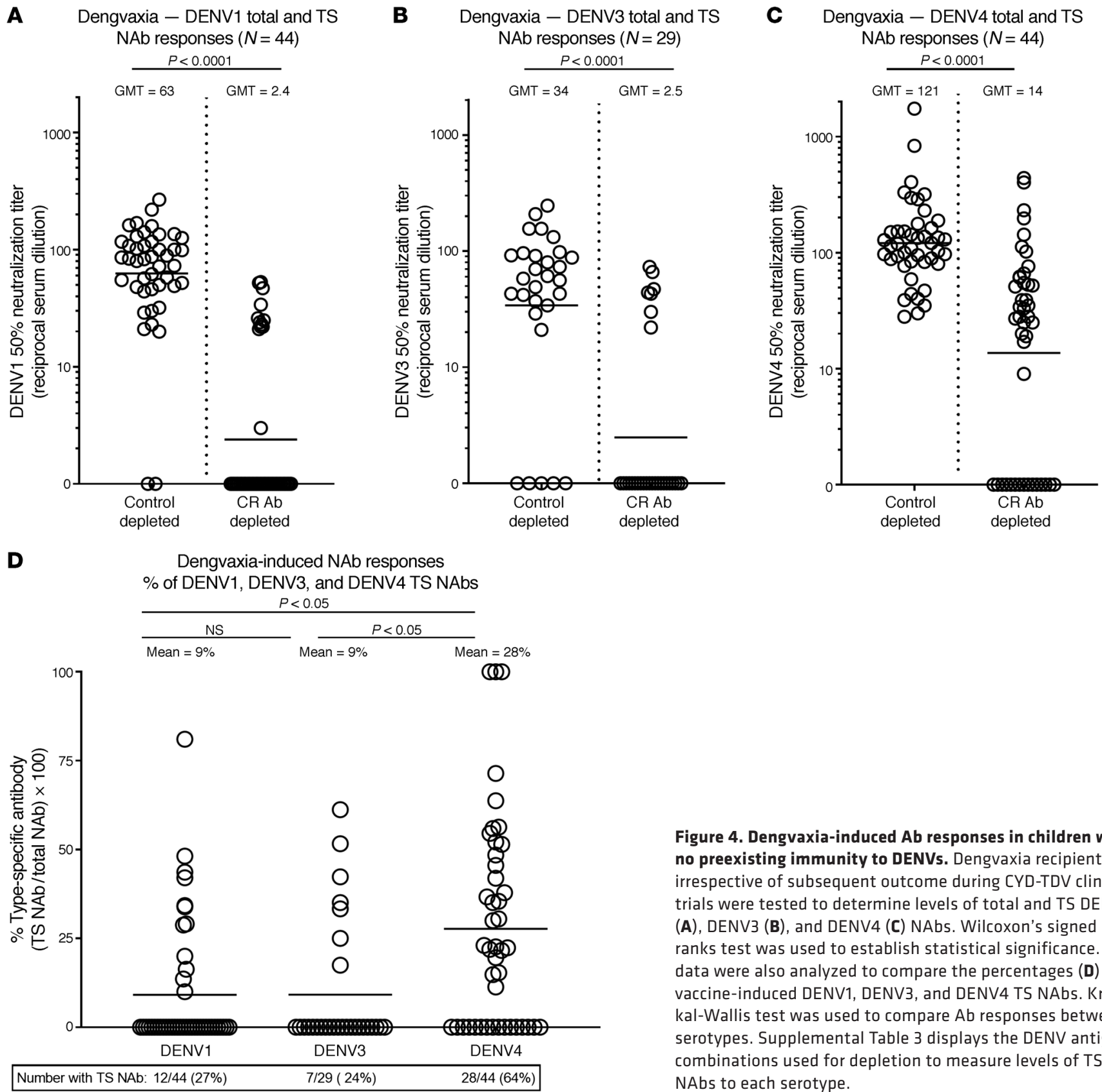

\begin{abstract}
Figure 4. Dengvaxia-induced Ab responses in children with no preexisting immunity to DENVs. Dengvaxia recipients irrespective of subsequent outcome during CYD-TDV clinical trials were tested to determine levels of total and TS DENV1 (A), DENV3 (B), and DENV4 (C) NAbs. Wilcoxon's signed ranks test was used to establish statistical significance. The data were also analyzed to compare the percentages (D) of vaccine-induced DENV1, DENV3, and DENV4 TS NAbs. Kruskal-Wallis test was used to compare Ab responses between serotypes. Supplemental Table 3 displays the DENV antigen combinations used for depletion to measure levels of TS NAbs to each serotype.
\end{abstract}

Institutional Review Board of the University of North Carolina at Chapel Hill reviewed and approved the receipt and analysis of anonymized CYD14 and CYD15 clinical specimens at the University of North Carolina at Chapel Hill (protocol 16-0793). The clinical specimens from individuals exposed to WT DENV infections were obtained from the UNC Arbovirus traveler study to recruit individuals in the US infected during foreign travel. The collection, storage, and use of these samples for research has been approved by the Institutional Review Board of the University of North Carolina at Chapel Hill (protocol 19-2187).

\section{Author contributions}

$\mathrm{MB}$ helped with the initial characterization and selection of clinical samples for this study. RB, AMDS, SH, CA, MB, JMM, and AM designed the research studies and assisted with the analysis of results. CA and $\mathrm{SH}$ conducted experiments and wrote the first drafts of the manuscript. All the authors provided critical edits.

\section{Acknowledgments}

This research was supported by US National Institute of Allergy and Infectious Diseases (NIAID) grants R01 AI107731 and R01 AI125198 (PI: AMDS).

Address correspondence to: Aravinda de Silva, UNC-Chapel Hill, CB\# 7292, 160 Dental Circle, 9024 Burnett Womack, Chapel Hill, North Carolina 27599-7292, USA. Phone: 919.962.4891; Email: desilva@med.unc.edu. 
1. Bhatt $\mathrm{S}$, et al. The global distribution and burden of dengue. Nature. 2013;496(7446):504-507.

2. de Alwis R, et al. In-depth analysis of the antibody response of individuals exposed to primary dengue virus infection. PLoS Negl Trop Dis. 2011;5(6):e1188.

3. Guzman MG, et al. Neutralizing antibodies after infection with dengue 1 virus. Emerg Infect Dis. 2007;13(2):282-286

4. Mathew A, et al. B-cell responses during primary and secondary dengue virus infections in humans. J Infect Dis. 2011;204(10):1514-1522.

5. Biswal S, et al. Efficacy of a tetravalent dengue vaccine in healthy children aged 4-16 years: a randomised, placebo-controlled, phase 3 trial. Lancet. 2020;395(10234):1423-1433.

6. Dayan GH, et al. Efficacy after 1 and 2 doses of CYD-TDV in dengue endemic areas by dengue serostatus. Vaccine. 2020;38(41):3472-6477.

7. Moodie Z, et al. Neutralizing antibody correlates analysis of tetravalent dengue vaccine efficacy trials in Asia and Latin America. J Infect Dis. 2018;217(5):742-753.

8. Sabin AB. Research on dengue during World War II. Am J Trop Med Hyg. 1952;1(1):30-50.

9. Halstead SB. In vivo enhancement of dengue virus infection in rhesus monkeys by passively transferred antibody. J Infect Dis. 1979;140(4):527-533.

10. Gibbons RV, et al. Analysis of repeat hospital admissions for dengue to estimate the frequency of third or fourth dengue infections resulting in admissions and dengue hemorrhagic fever, and serotype sequences. Am J Trop Med Hyg. 2007;77(5):910-913.
11. Olkowski S, et al. Reduced risk of disease during postsecondary dengue virus infections. Infect Dis. 2013;208(6):1026-1033.

12. Thomas SJ, Yoon IK. A review of Dengvaxia: development to deployment. Hum Vaccin Immunother. 2019;15(10):2295-2314.

13. Arredondo-Garcia JL, et al. Four-year safety follow-up of the tetravalent dengue vaccine efficacy randomized controlled trials in Asia and Latin America. Clin Microbiol Infect. 2018;24(7):755-763.

14. Hadinegoro SR, et al. Efficacy and long-term safety of a dengue vaccine in regions of endemic disease. N Engl J Med.2015;373(13):1195-1206.

15. Sabchareon A, et al. Protective efficacy of the recombinant, live-attenuated, CYD tetravalent dengue vaccine in Thai schoolchildren: a randomised, controlled phase $2 \mathrm{~b}$ trial. Lancet . 2012;380(9853):1559-1567.

16. Sridhar S, et al. Effect of dengue serostatus on dengue vaccine safety and efficacy. $N$ Engl J Med 2018;379(4):327-340

17. Gallichotte EN, et al. The molecular specificity of the human antibody response to dengue virus infections. Adv Exp Med Biol. 2018;1062:63-76.

18. Tsai WY, et al. Potent neutralizing human monoclonal antibodies preferentially target mature dengue virus particles: implication for novel strategy for dengue vaccine. J Virol. 2018;92(23): $000556-18$

19. Henein S, et al. Dissecting antibodies induced by a chimeric yellow fever-dengue, live-attenuated, tetravalent dengue vaccine (CYD-TDV) in naive and dengue-exposed individuals. J Infect Dis. 2017;215(3):351-358 .
20. Barban V, et al. Improvement of the dengue virus (DENV) nonhuman primate model via a reverse translational approach based on dengue vaccine clinical efficacy data against DENV-2 and -4. JVirol. 2018;92(12):e00440-18.

21. Torresi J, et al. Replication and excretion of the live attenuated tetravalent dengue vaccine CYD-TDV in a flavivirus-naive adult population: assessment of vaccine viremia and virus shedding. J Infect Dis. 2017;216(7):834-841.

22. Carpp LN, et al. Microneutralization assay titer correlates analysis in two phase 3 trials of the CYD-TDV tetravalent dengue vaccine in Asia and Latin America. PLoS One. 2020;15(6):e0234236.

23. Raut $\mathrm{R}$, et al. Dengue type 1 viruses circulating in humans are highly infectious and poorly neutralized by human antibodies. Proc Natl Acad Sci US A. 2019;116(1):227-232.

24. Mukherjee S, et al. Pseudo-infectious reporter virus particles for measuring antibody-mediated neutralization and enhancement of dengue virus infection. Methods Mol Biol. 2014;1138:75-97.

25. Tsai WY, et al. High-avidity and potently neutralizing cross-reactive human monoclonal antibodies derived from secondary dengue virus infection. J Virol. 2013;87(23):12562-12575.

26. Capeding MR, et al. Clinical efficacy and safety of a novel tetravalent dengue vaccine in healthy children in Asia: a phase 3, randomised, observer-masked, placebo-controlled trial. Lancet. 2014;384(9951):1358-1365.

27. Villar L, et al. Efficacy of a tetravalent dengue vaccine in children in Latin America. $N$ Engl J Med. 2015;372(2):113-123. 